\title{
C5a promotes the proliferation of human nasopharyngeal carcinoma cells through PCAF-mediated STAT3 acetylation
}

\author{
KEMIN CAI $^{1 *}$, YI WAN $^{2 *}$, ZHIMIN WANG $^{2 *}$, YU WANG $^{1 *}$, XIAOJUN ZHAO $^{1 *}$ and XUELI BAO ${ }^{1}$ \\ ${ }^{1}$ Department of Otorhinolaryngology Head and Neck Surgery, Taizhou People's Hospital, \\ Taizhou, Jiangsu 225300; ${ }^{2}$ Department of Neurosurgery, Suzhou Kowloon Hospital Affiliated with \\ Shanghai Jiao Tong University School of Medicine, Suzhou, Jiangsu 215021, P.R. China
}

Received May 30, 2014; Accepted July 29, 2014

DOI: $10.3892 /$ or.2014.3420

\begin{abstract}
The anaphylatoxin $\mathrm{C} 5 \mathrm{a}$ is a chemoattractant that can induce various inflammatory responses in vivo via the C5a receptor $(\mathrm{C} 5 \mathrm{aR})$. There is emerging evidence that $\mathrm{C} 5 \mathrm{a}$ is generated in the cancer microenvironment. However, the role of $\mathrm{C} 5 \mathrm{a}$ in human nasopharyngeal carcinoma (NPC) remains largely unclear. Thus, the present study aimed to examine the direct influence of C5a stimulation on the proliferation of human NPC cells and to identify the underlying molecular mechanisms. The effects of C5a stimulation on the proliferation of human NPC cells were studied in vitro, and P300/CBP-associated factor (PCAF)-mediated signal transducer and activator of transcription 3 (STAT3) acetylation and its role in regulating the proliferation of NPC cells was subsequently explored. Our results demonstrated that $\mathrm{C} 5 \mathrm{a}$ stimulation increased the proliferation of human NPC cells in vitro. STAT3 acetylation was further found to be enhanced in human NPC cells induced by C5a. Moreover, PCAF induction was required for STAT3 acetylation in human NPC cells by exposure to C5a. Functionally, PCAF-mediated STAT3 acetylation contributed to the proliferation of human NPC cells stimulated by C5a. These results illustrate the novel activity of the C5a-C5aR axis that promotes human NPC cell proliferation through PCAF-mediated STAT3 acetylation. This may provide a potential strategy for treating human NPC through inhibition of C5a or its receptors.
\end{abstract}

\section{Introduction}

Nasopharyngeal carcinoma (NPC) is a common tumor in the head and neck $(1,2)$. NPC is one of the most common

Correspondence to: Dr Xueli Bao, Department of Otorhinolaryngology Head and Neck Surgery, Taizhou People's Hospital, Taizhou, Jiangsu 225300, P.R. China

E-mail: baoxuelichina@163.com

*Contributed equally

Key words: nasopharyngeal carcinoma, proliferation, C5a, P300/ CBP-associated factor, signal transducer and activator of transcription 3, acetylation malignancies in Southern China and Southeast Asia with an incidence rate of 20-30 cases per 100,000 individuals $(3,4)$, and is believed to be associated with EB virus infection $(4,5)$. However, its pathogenesis remains unclear. It is known that tumor development is a multistep process of cumulative genetic alterations that lead to cell autonomy. During this process, inflammatory mechanisms are thought to play a critical role $(6,7)$.

Complement activation is believed to play a critical role in inflammatory responses in vivo (8). The recent finding that complement may contribute to tumor growth suggests an insidious relationship between complement and cancer, particularly in light of evidence that complement facilitates cellular proliferation and regeneration $(9,10)$. During carcinogenesis, tumor cells acquire genetic and epigenetic alterations that dictate their malignant growth. Because of these alterations, the complement system can recognize tumor cells, as can be shown by the complement deposition found in different tumors. In fact, new findings on the roles of complement in tumor growth have challenged the recognition that complement always protects against tumors. Some studies have demonstrated that the generation of complement especial anaphylatoxin $\mathrm{C} 5 \mathrm{a}$ in the tumor microenvironment leads to significant tumor progression, such as lung cancer (9-11).

Current research has revealed that the signal transducer and activator of transcription 3 (STAT3) plays a pivotal role in NPC development. Activation of STAT3 may contribute to both the development and progression of NPC (12-14). Thus, targeting aberrant STAT3 signaling may provide an effective and novel strategy for the treatment of NPC (15). Despite the fact that STAT3 activation is common in NPC, the mechanism of STAT3 activation in NPC has not been fully elucidated.

It is well known that protein function is often regulated by post-translational modifications such as acetylation $(16,17)$, and mounting evidence suggests that protein acetylation plays important roles in various biological events including transcriptional regulation, DNA damage repair, cell proliferation and autophagy (18-22). Many studies have revealed that signaling molecule STAT3 undergoes phosphorylation, which is necessary for STAT3 activation in various diseases (23). However, the STAT3 acetylation and its roles in regulating STAT3 activation in NPC remain largely unclear. 
Lysine (K) acetyltransferase $2 \mathrm{~B}$, also known as P300/CBP-associated factor (PCAF), is a trancriptional co-activator, which has been shown to interact with Myc, $\beta$-catenin, Homeobox A10 (HOXA10) and histone (24-28). PCAF has acetyl transferase activity with various transcription factors and signaling molecules (28-31), indicating that PCAF may play direct roles in regulating the activity of transcription factors and signaling molecules. PCAF has also been reported to be related to cancer (32). However, the roles of PCAF in mediating STAT3 activation and the proliferation of NPC need to be explored.

In the present study, we evaluated the implication of C5a in the proliferation of human NPC cells in vitro. PCAF-mediated STAT3 acetylation and its role in regulating the proliferation of NPC cells were subsequently explored. Our results revealed that C5a stimulated the proliferation of human NPC cells in vitro. The level of STAT3 acetylation was elevated in human NPC cells induced by C5a. Moreover, PCAF induction was required for STAT3 acetylation in human NPC cells by exposure to C5a. Functionally, PCAF-mediated STAT3 acetylation contributed to the proliferation of human NPC cells stimulated by $\mathrm{C} 5 \mathrm{a}$. Taken together, our findings suggest that $\mathrm{C} 5 \mathrm{a}$ promotes the proliferation of NPC cells through PCAF-mediated STAT3 acetylation.

\section{Materials and methods}

Reagents. Monoclonal antibodies against human PCAF were obtained from Santa Cruz Biotechnology (Santa Cruz, CA, USA). Monoclonal antibodies against human $\beta$-actin, STAT3, phospho-STAT3 (Tyr705), phospho-STAT3 (Ser727) and acetylated-lysine were from Cell Signaling Technology (Danvers, MA, USA). For the western blot analysis, horseradish peroxidase-conjugated anti-mouse $\mathrm{IgG}$ antibody, $20 \mathrm{X}$ LumiGLO reagent and $20 \mathrm{X}$ peroxide were purchased from Cell Signaling Technology. PVDF membranes were from Millipore (Billerica, MA, USA). TRIzol reagent was purchased from Invitrogen (Carlsbad, CA, USA). MMLV was provided by Promega (Madison, WI, USA). A RevertAid ${ }^{\mathrm{TM}}$ First Strand cDNA Synthesis kit was from Fermentas (Pittsburgh, PA, USA). TaqMan ${ }^{\circledR}$ Fast Advanced Master Mix was from ABI (Foster, CA, USA). The pGCsi.U6.neo.GFP plasmid was obtained from Shanghai Genkan Biotechnology Co., Ltd. (Shanghai, China). The pcDNA3.1 vector was from Invitrogen. The incision enzymes HindIII and BamHI as well as T4 DNA ligase were purchased from Takara (Tokyo, Japan). A QIAprep Spin Miniprep kit was obtained from Qiagen (Hilden, Germany). Human complement 5a (C5a) was obtained from R\&D Systems (Minneapolis, MN, USA). Cell Counting Kit-8 (CCK-8) was purchased from Dojindo Laboratories (Kumamoto, Japan).

Cell culture. The human NPC cell lines, CNE1 and C666-1, and the control human nasopharyngeal epithelial cell line, NP69, were obtained from the American Type Culture Collection (ATCC, Manassas, VA, USA). Cell lines was cultured in RPMI-1640 medium (Gibco, Carlsbad, CA, USA) supplemented with $10 \%$ (v/v) fetal bovine serum (FBS; Gibco) and antibiotics $(50 \mathrm{U} / \mathrm{ml}$ penicillin and $100 \mu \mathrm{g} / \mathrm{ml}$ streptomycin; Invitrogen, Carlsbad, CA, USA) at $37^{\circ} \mathrm{C}$ in $5 \% \mathrm{CO}_{2}$.
Generation of the overexpression plasmids. The pcDNA3.1/ PCAF-His plasmids were constructed by inserting the ORF of human PCAF (NM_003884.4) cDNA into the mammalian expression plasmids of pcDNA3.1. The PCAF gene containing a His tag was amplified by polymerase chain reaction (PCR) from cDNA of normal human nasopharyngeal epithelial cells. The PCR products and pcDNA3.1 vector were further digested with the two restriction enzymes, HindIII and BamHI, and then ligated by using T4 DNA ligase. The recombinant plasmids were amplified in E.coli strain $\mathrm{DH} 5 \alpha$ and purified with a QIAprep Spin Miniprep kit. Finally, the constructed plasmids were sequenced across both junctions to confirm the nucleotide sequence and the predicted orientation.

Construction of the shRNA expression plasmids. To silence the PCAF gene in human NPC cells, there different shRNA sequences against human PCAF mRNA (NM_003884.4) were designed. The different DNA segments of PCAF shRNA were constructed into the pGCsi.U6.neo.GFP plasmids, and the most effective shRNA expression plasmid was chosen for further functional experiments.

Cellular transfection. NPC cell lines were transfected using Lipofectamine 2000 according to the manufacturer's instructions. Briefly, $4 \mu \mathrm{g}$ of the plasmids was mixed with $250 \mu \mathrm{l}$ of serum-free medium, and then $10 \mu \mathrm{l}$ of Lipofectamine 2000 mixed with $250 \mu \mathrm{l}$ of serum-free medium was added and incubated for $20 \mathrm{~min}$ at room temperature. Finally, the resultant mixture was added to the cells in each well. The medium was replaced with serum-containing medium $5 \mathrm{~h}$ after transfection $(33,34)$.

Co-immunoprecipitation experiment. Three hundred micrograms of extract, prepared from the NPC cell lines (CNE1 and C666-1) and the normal nasopharyngeal epithelial cell line (NP69) was mixed with $40 \mu 1$ protein G-Sepharose beads in co-IP assay buffer, incubated for $2 \mathrm{~h}$ and centrifuged for $2 \mathrm{~min}$. The recovered supernatant was then incubated with the corresponding antibody $(2 \mu \mathrm{g}$, preimmune $\mathrm{IgG}$ as a control reaction) at $4^{\circ} \mathrm{C}$ overnight. Then, $40 \mu \mathrm{l}$ of protein G-Sepharose beads was added, and incubation was continued for $2.5 \mathrm{~h}$. Protein G-precipitated protein complex was recovered by centrifugation and harvested beads resuspended in $30 \mu \mathrm{l}$ of $2 \mathrm{X}$ SDS-PAGE sample buffer were boiled for $5 \mathrm{~min}$. The samples were then analyzed by western blot analysis with specific antibodies. A 40- $\mu$ g aliquot of whole-cell extract (WCE) was used as an input control (35).

RNA isolation and real-time quantitative PCR. The total RNA was isolated from the cells using TRIzol reagent. An equal amount $(4 \mu \mathrm{g})$ of total RNA was synthesized as a first-strand cDNA using the RevertAid ${ }^{\mathrm{TM}}$ First Strand cDNA Synthesis kit. The cDNA was amplified using TaqMan ${ }^{\circledR}$ Fast Advanced Master Mix to detect the expression of the PCAF gene. The reaction program consisted of an initial denaturation step at $95^{\circ} \mathrm{C}$ for $10 \mathrm{~min}$, denaturation at $95^{\circ} \mathrm{C}$ for $15 \mathrm{sec}$ and annealing at $60^{\circ} \mathrm{C}$ for $60 \mathrm{sec}$ for 40 cycles. The 7500 Real-time PCR system (ABI) was used for the experiment. Each sample was assayed in triplicate. The $\beta$-actin gene was used as an internal control. The relative level of gene expression was obtained by 
A

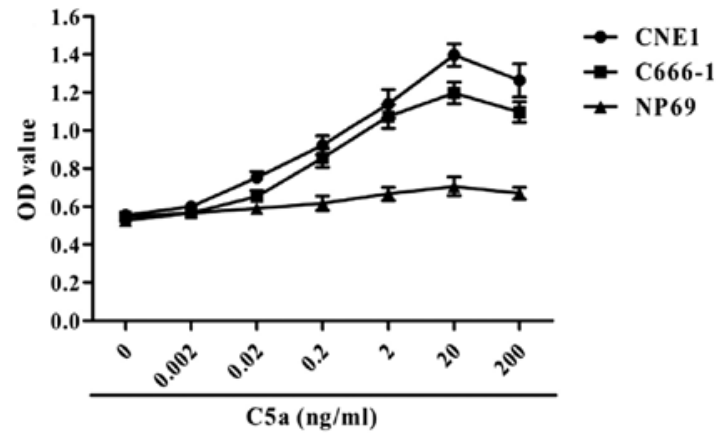

B

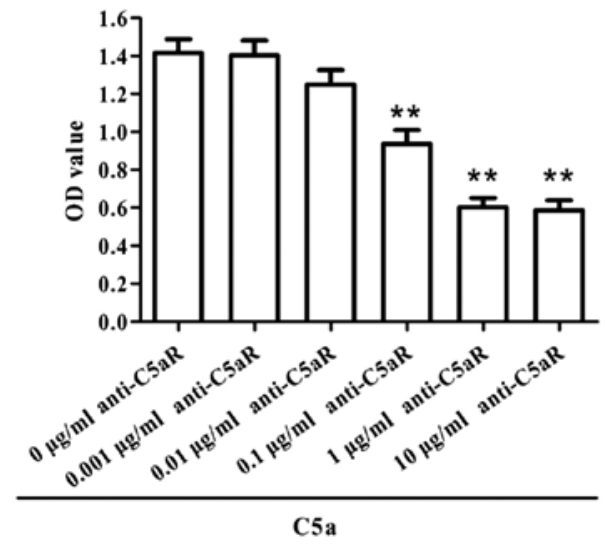

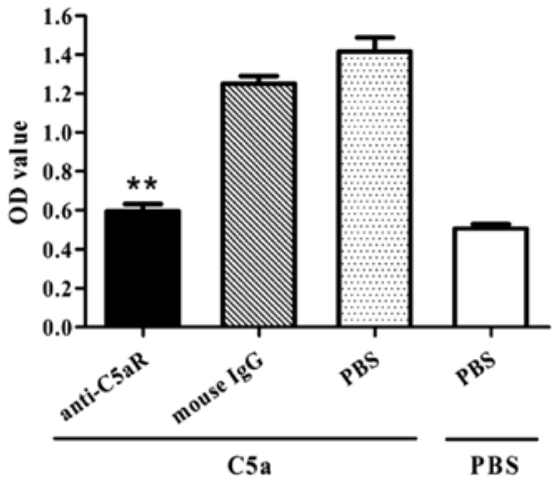

D

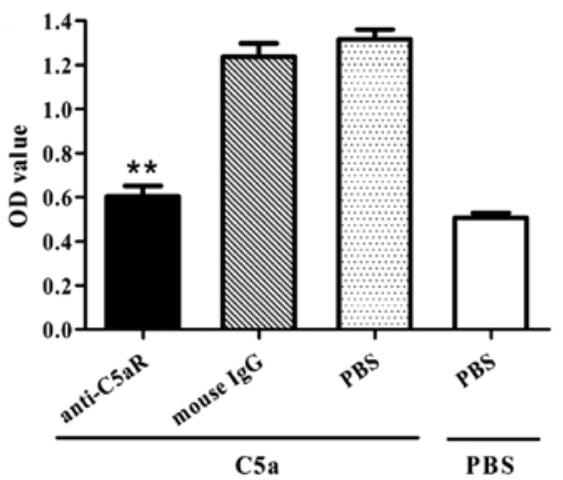

Figure 1. Effect of C5a on the proliferation of human NPC cells. (A) Different concentrations of purified human C5a were added to human NPC cell lines (CNE1 and C666-1) and a normal human nasopharyngeal epithelial cell line (NP69) as a control to observe its ability to induce the proliferation of NPC cells. CCK-8 analysis showed that C5a stimulation for $48 \mathrm{~h}$ enhanced the proliferation of both human NPC cell lines in a dose-dependent manner in vitro, but had no marked effect on the proliferation of the NP69 cells. (B) Different concentrations of anti-C5aR neutralizing antibodies were added to the media of the CNE1 cell line $30 \mathrm{~min}$ before adding C5a $(20 \mathrm{ng} / \mathrm{ml})$, and then the proliferation of the CNE1 cells induced by C5a for $48 \mathrm{~h}$ was detected. CCK-8 analysis showed that blockade of the C5a receptor suppressed the C5a-triggered proliferation of the CNE1 cell line in a dose-dependent manner. There was no significant difference between the $1 \mu \mathrm{g} / \mathrm{ml}$ and $10 \mu \mathrm{g} / \mathrm{ml}$ dose. ${ }^{* *} \mathrm{P}<0.01 \mathrm{vs} .0 \mu \mathrm{g} / \mathrm{ml}+\mathrm{C} 5 \mathrm{a}$ group. (C and D) Anti-C5aR neutralizing antibodies (1 $\left.\mu \mathrm{g} / \mathrm{ml}\right)$ were added to the media of NPC cell lines (C, CNE1; D, C666-1) $30 \mathrm{~min}$ before adding C5a (20 ng/ml), and then the proliferation of the NCP cell lines induced by C5a for $48 \mathrm{~h}$ was evaluated. CCK-8 analysis showed that blockade of the C5a receptor completely reduced the $\mathrm{C} 5 \mathrm{a}$-triggered proliferation of both $\mathrm{NPC}$ cell lines. ${ }^{* *} \mathrm{P}<0.01$ vs. PBS + C5a group and mouse IgG + C5a group. The data are from one experiment, and are representative of three independent experiments. Results are expressed as means $\pm \mathrm{SD}$.

calculating the ratio of cycle numbers of the initial exponential amplification phase as determined by the sequence detection system for the specific target gene and $\beta$-actin using the following formula: $2^{-\Delta \Delta C \mathrm{t}}$.

Western blot analysis. The proteins $(40 \mu \mathrm{g})$ were subjected to $10-15 \%$ SDS polyacrylamide gel electrophoresis and transferred onto PVDF membranes by the Mini-Protean System (Bio-Rad, Hercules, CA, USA). The membranes were incubated for $1 \mathrm{~h}$ at room temperature (RT) in blocking buffer (5\% skimmed milk in TBS-T) and then incubated with the appropriate antibodies overnight at $4^{\circ} \mathrm{C}$. After washing with TBST-T, the membranes were incubated with horseradish peroxidase-conjugated anti-rabbit and anti-mouse for $1 \mathrm{~h}$ at $37^{\circ} \mathrm{C}$. The bands were visualized by the ECL detection system with 3-10 min exposure after washing the membranes. The radiographic band density was measured using Quantity One ${ }^{\circledR}$ software (Bio-Rad). Control for protein loading was identified by $\beta$-actin as the internal standard. Each sample was assayed in triplicate.

$C C K-8$ assay. The NPC cell lines and the normal human nasopharyngeal epithelial cell line after the different treat- ments were incubated with CCK- 8 for a final $4 \mathrm{~h}$. The formazan product was visualized at an absorbance of $450 \mathrm{~nm}$, and the absorbance was directly proportional to the cell number $(35,36)$. Each sample was assayed in triplicate.

Statistical analysis. All statistical analyses were carried out using SPSS ${ }^{\circledR} 11.5$ software. All data are expressed as mean $\pm \mathrm{SD}$. The statistical significance (defined as $\mathrm{P}<0.05$ ) of the groups was evaluated by one-way ANOVA with simultaneous multiple comparisons between groups by the Bonferroni method.

\section{Results}

C5a promotes the proliferation of human NPC cells. Purified human C5a was added to the human NPC cell lines (CNE1 and C666-1) to observe its ability to induce the proliferation of NPC cells. The in vitro studies showed that C5a enhanced the proliferation of both human NPC cell lines in a dose-dependant manner, but had no marked effect on the proliferation of the normal human nasopharyngeal epithelial cell line (Fig. 1A). In order to confirm whether this was due to the effect of C5a, anti-C5aR neutralizing antibodies were added to the media of 

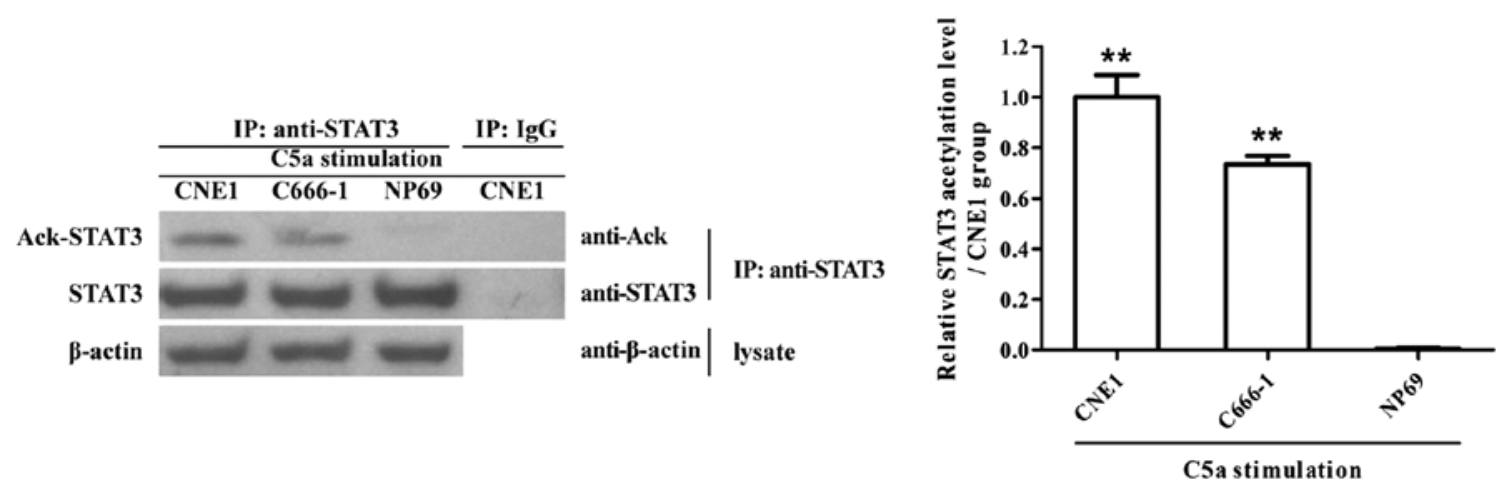

Figure 2. Effect of C5a on STAT3 acetylation in human NPC cells. CNE1, C666-1 and NP69 cell lines were stimulated with C5a (20 ng/ml) for $3 \mathrm{~h}$, and antiSTAT3 antibodies were used to carry out immunoprecipitation. Subsequently, acetylated STAT3 (Ack-STAT3) and total STAT3 in the immunoprecipitation complex were determined by western blot analysis. The results show that the level of STAT3 acetylation in both human NPC cell lines induced by C5a was significantly higher than that in the NP69 cell line. The data are from one experiment, and are representative of three independent experiments. Results are expressed as means $\pm \mathrm{SD}$. Representative western blotting images are shown. ${ }^{* *} \mathrm{P}<0.01$ vs. the NP69 group.

A

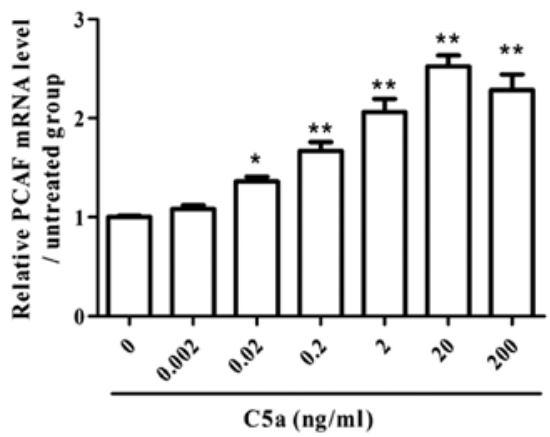

B

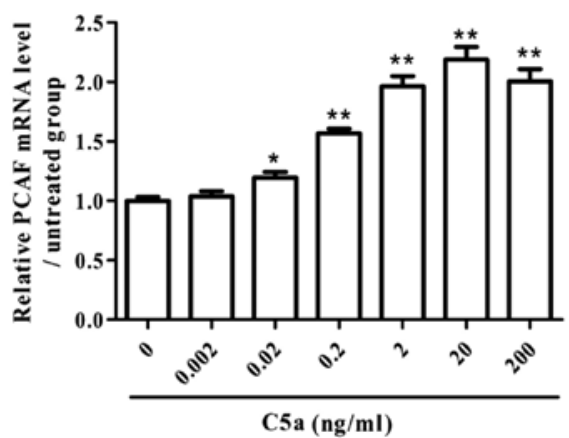

C

\begin{tabular}{cccc}
\multicolumn{2}{c}{ IP: anti-STAT3 } & IP: IgG \\
\cline { 1 - 3 } & C5a stimulation & \\
\hline CNE1 & C666-1 & NP69 & CNE1
\end{tabular}
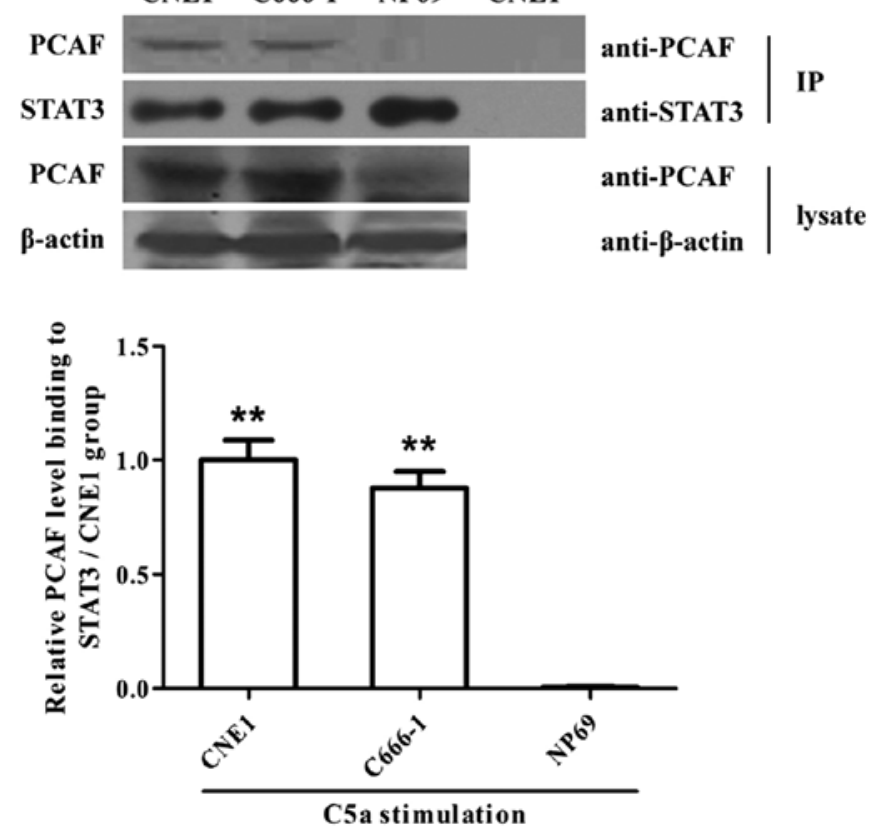

Figure 3. The role of C5a in PCAF expression and its association with STAT3 in human NPC cells. (A and B) The expression of PCAF at the mRNA level was detected in human NPC cell lines stimulated by C5a for $3 \mathrm{~h}$, and real-time quantitative PCR showed that C5a markedly enhanced the expression levels of PCAF mRNA in both human NPC cell lines (A, CNE1; B, C666-1) relative to the normal human nasopharyngeal epithelial cell line (NP69). "P<0.05; ${ }^{* *} \mathrm{P}<0.01$ vs. the untreated group $(0 \mathrm{ng} / \mathrm{ml})$. (C) Co-immunoprecipitation analysis showed the interaction of PCAF with STAT3 at the protein level in both human NPC cell lines (CNE1 and C666-1) in response to C5a stimulation for $3 \mathrm{~h}$ was stronger than that in the normal human nasopharyngeal epithelial cell line (NP69). ${ }^{* *} \mathrm{P}<0.01$ vs. the NP69 group. The data are from one experiment, and are representative of three independent experiments. Results are expressed as means \pm SD. Representative western blotting images are shown.

NPC cells 30 min before adding C5a, and the proliferation of NCP cells induced by C5a was subsequently evaluated. The data showed that blockade of the C5a receptor completely reduced the C5a-triggered NPC cell proliferation (Fig. 1B-D). These findings indicate that anaphylatoxin C5a promoted the proliferation of human NPC cell lines but not that of the normal nasopharyngeal epithelial cell line.

STAT3 acetylation is enhanced in human NPC cells induced by $\mathrm{C} 5 \mathrm{a}$. Since C5a was found to stimulate human NPC cell proliferation (Fig. 1A-C), and STAT3 activation is believed to be involved in human NPC cell proliferation $(12,13)$, the effect of C5a stimulation on STAT3 acetylation was subsequently determined in NPC cells exposed to C5a. We found that the level of STAT3 acetylation was significantly enhanced in both human NPC cell lines induced by C5a in vitro, while the expression of STAT3 was not altered (Fig. 2). On the other hand, the results showed that the level of STAT3 phosphorylation was also increased in the human NPC cells following exposure to C5a (Fig. 4A). 
A
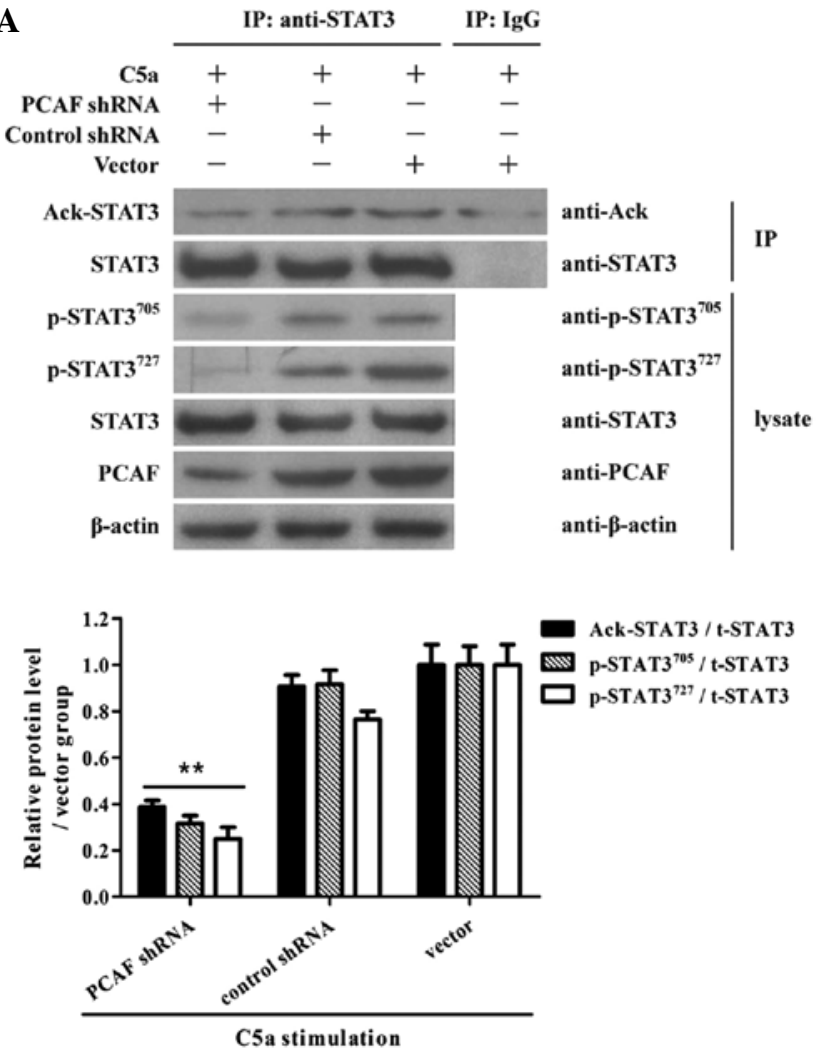

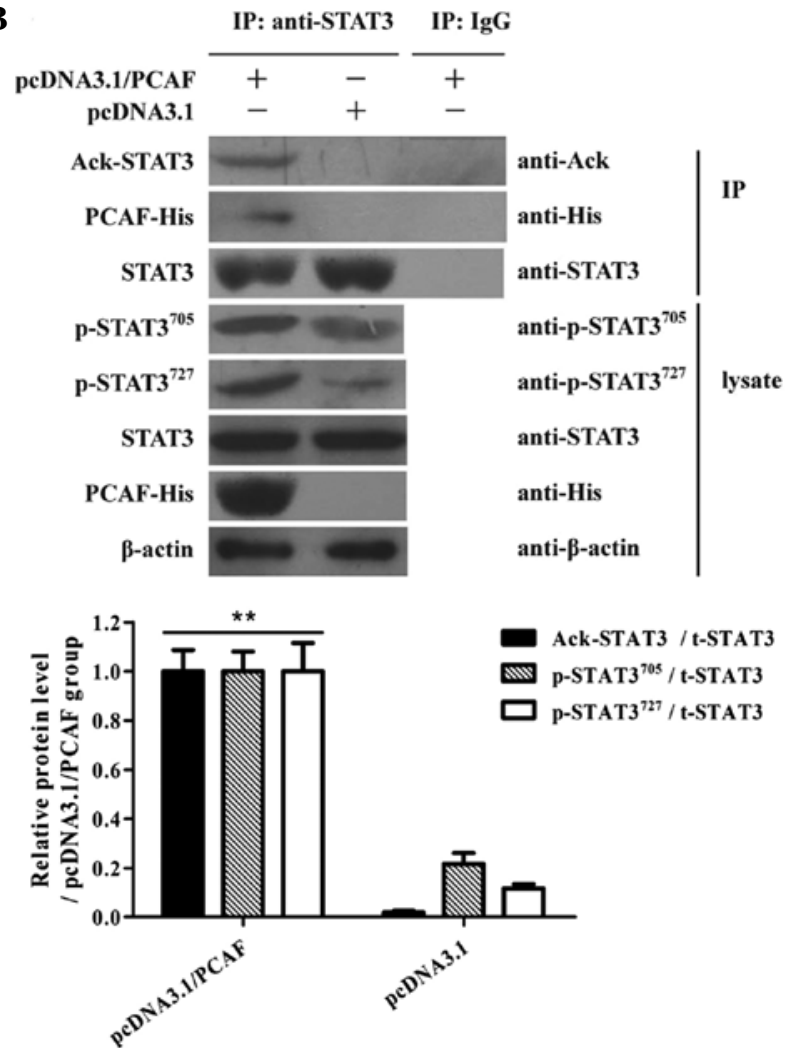

Figure 4. The role of PCAF induction in STAT3 acetylation in NPC cells following exposure to C5a. (A) CNE1 cells were treated with PCAF shRNA for $48 \mathrm{~h}$ followed by C5a stimulation for $3 \mathrm{~h}$. Co-immunoprecipitation assay showed that PCAF shRNA suppressed STAT3 acetylation and phosphorylation in CNE1 cells by exposure to C5a. ${ }^{* *} \mathrm{P}<0.01$ vs. control shRNA + C5a group and vector + C5a group. (B) Co-immunoprecipitation analysis revealed that overexpression of PCAF using pcDNA3.1/PCAF-His for $48 \mathrm{~h}$ elevated STAT3 acetylation and phosphorylation in CNE1 cells relative to the pcDNA3.1 vector group. ${ }^{* *} \mathrm{P}<0.01$ vs. the pcDNA3.1 group. The data are from one experiment, and are representative of three independent experiments. Results are expressed as means \pm SD. Representative western blotting images are shown.

A

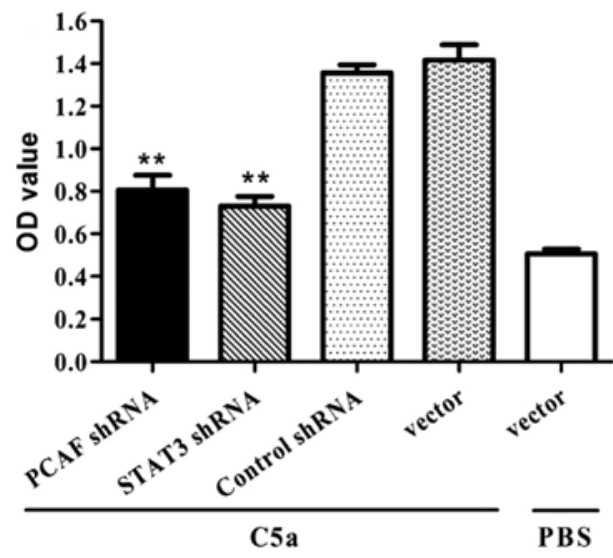

B

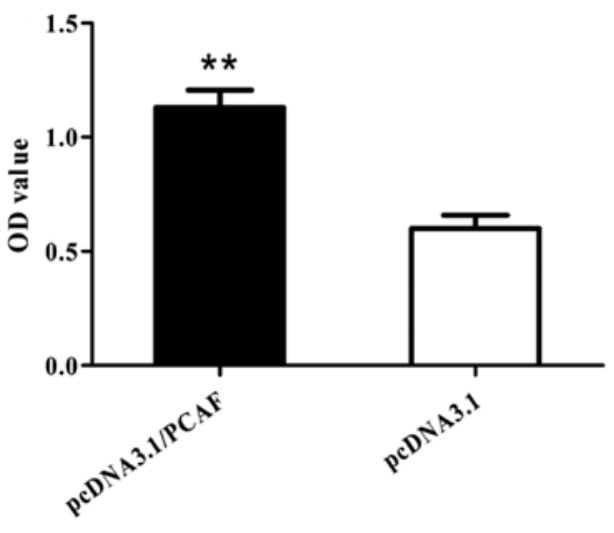

Figure 5. The role of PCAF-mediated STAT3 acetylation in the proliferation of NPC cells stimulated by C5a. (A) CNE1 cells were treated with PCAF shRNA for $48 \mathrm{~h}$ followed by C5a stimulation for $48 \mathrm{~h}$. CCK-8 assay showed that suppression of PCAF or STAT3 using shRNA pretreatment inhibited the cellular proliferation of CNE1 cells induced by C5a. ${ }^{* *} \mathrm{P}<0.01$ vs. control shRNA + C5a group and vector + C5a group. (B) CCK-8 analysis revealed that CNE1 cells overexpressing PCAF for $72 \mathrm{~h}$ exhibited enhanced cellular proliferation compared to the pcDNA3.1 vector group. ${ }^{* *} \mathrm{P}<0.01 \mathrm{vs}$. the pcDNA3.1 group. The data are from one experiment, and are representative of three independent experiments. Results are expressed as means \pm SD.

PCAF expression and its association with STAT3 are increased in human NPC cells exposed to C5a. The expression of PCAF at both the mRNA and protein levels was detected in human NPC cells stimulated by C5a, and we found that C5a markedly enhanced the expression levels of PCAF mRNA and protein in both human NPC cell lines (Fig. 3A and B).
The interaction of PCAF with STAT3 at the protein level was also found to be enhanced in both human NPC cell lines in response to $\mathrm{C} 5 \mathrm{a}$ stimulation relative to the normal human nasopharyngeal epithelial cell line (Fig. 3B). These findings indicate that $\mathrm{C} 5 \mathrm{a}$ stimulation induced the expression of PCAF and further enhanced the interaction of PCAF with STAT3 at 
the protein level in human NPC cells, suggesting a potential role of PCAF in acetylating STAT3.

PCAF induction is required for STAT3 acetylation in NPC cells by exposure to $C 5 a$. In order to test the requirement of PCAF expression in STAT3 acetylation and phosphorylation, CNE1 cells were treated with PCAF shRNA for $48 \mathrm{~h}$ followed by $\mathrm{C} 5$ a stimulation. Co-immunoprecipitation assay showed that shRNA depletion of PCAF suppressed STAT3 acetylation and phosphorylation in the NPC cells following exposure to C5a (Fig. 4A). Furthermore, overexpression of PCAF elevated STAT3 acetylation and phosphorylation in NPC cells (Fig. 4B). Taken together, these data suggest that acetyltransferase activity of PCAF is required for Aktl acetylation and phosphorylation in NPC cells.

PCAF-mediated STAT3 acetylation contributes to the proliferation of NPC cells stimulated by $C 5 a$. Since PCAF induction was found to be required for STAT3 acetylation in NPC cells by exposure to C5a (Fig. 4), we further explored the role of PCAF-mediated STAT3 acetylation in the proliferation of NPC cells stimulated by C5a. The results showed that suppression of PCAF or STAT3 could cause significant inhibition with regards to cellular proliferation in NPC cells induced by $\mathrm{C} 5 \mathrm{a}$ (Fig. 5A). Meanwhile, NCP cells overexpressing PCAF exhibited enhanced cellular proliferation (Fig. 5B). Taken together, these data indicate that PCAF-mediated STAT3 acetylation contributes to the proliferation of NPC cells stimulated by C5a.

\section{Discussion}

Nasopharyngeal carcinoma (NPC) is a common tumor in the head and neck, and is one of the most common malignancies in Southern China and Southeast Asia (1-4). However, the molecular pathogenesis of NPC remains largely unclear. Recently, inflammatory responses have been thought to play a pivotal role in the process of cancer development $(6,7)$. Complement activation particularly C5a generation is believed to play a critical role in regulating inflammatory responses in vivo $(8,37,38)$, and contribute to cancer progression (9-11). Our present studies showed that $\mathrm{C5a}$ in vitro enhanced the proliferation of human NPC cells, but had no significant influence on the proliferation of normal human nasopharyngeal epithelial cells. Anti-C5aR neutralizing antibodies were added to the culture media of NPC cells 30 min before adding C5a, and the proliferation assay showed that blockade of the C5a receptor completely reduced the C5a-triggered NPC cell proliferation. These findings indicate that overproduction of C5a may contribute to the proliferation of human NPC cells in vivo.

Signal transducer and activator of transcription 3 (STAT3) was found to be activated in the majority of NPC patients and is clinically correlated with advanced disease (stages III and IV). Therefore, activation of STAT3 may contribute to both the development and progression of NPC $(14,39,40)$. Thus, targeting aberrant STAT3 signaling may provide an effective and novel strategy for the treatment of NPC $(13,15)$. Despite the fact that STAT3 activation is common in NPC, the mechanisms of STAT3 activation in NPC have not been fully elucidated. Given the importance of STAT3 activation and C5a-triggered proliferation in NPC pathogenesis (Fig. 1), we examined the effect of C5a stimulation on STAT3 acetylation in NPC cells. Our data showed that the level of STAT3 acetylation was significantly elevated in human NPC cells induced by $\mathrm{C} 5 \mathrm{a}$.

Further experiments were designed to explore the mechanism of STAT3 acetylation. It is well accepted that PCAF has acetyl transferase activity and plays direct roles in regulating the activity of transcription factors and signaling molecules (24-28). However, the roles of P300/CBP-associated factor (PCAF) in mediating STAT3 activation as well as the proliferation of NPC are largely unclear. Our research revealed that PCAF silencing suppressed STAT3 acetylation and phosphorylation in NPC cells in response to C5a. In contrast, overexpression of PCAF elevated STAT3 acetylation and phosphorylation in NPC cells. Subsequent experiments showed that suppression of PCAF or STAT3 inhibited the proliferation of NPC cells induced by C5a, while overexpression of PCAF in NCP cells enhanced cellular proliferation.

In summary, in the present study, we evaluated the implication of C5a in the proliferation of human NPC cells in vitro. C5a-induced PCAF expression and PCAF-mediated STAT3 acetylation as well as their roles in regulating the proliferation of NPC cells were explored. Collectively, our findings suggest that C5a promotes the proliferation of NPC cells through PCAF-mediated STAT3 acetylation. These may provide a potential strategy for treating human NPC through inhibition of C5a or its receptors.

\section{References}

1. Yan $\mathrm{M}$, Zhang $\mathrm{Y}, \mathrm{He} \mathrm{B}$, et al: IKK $\alpha$ restoration via EZH2 suppression induces nasopharyngeal carcinoma differentiation. Nature Commun 5: 3661, 2014.

2. Du XJ, Tang LL, Mao YP, et al: The pretreatment albumin to globulin ratio has predictive value for long-term mortality in nasopharyngeal carcinoma. PloS One 9: e94473, 2014.

3. Wee J, Ha TC, Loong S and Qian CN: High incidence of nasopharyngeal cancer: similarity for $60 \%$ of mitochondrial DNA signatures between the Bidayuhs of Borneo and the Bai-yue of Southern China. Chin J Cancer 31: 455-456, 2012.

4. Han BL, Xu XY, Zhang CZ, et al: Systematic review on Epstein-Barr virus (EBV) DNA in diagnosis of nasopharyngeal carcinoma in Asian populations. Asian Pac J Cancer Prev 13: 2577-2581, 2012.

5. Feng $\mathrm{X}$, Ching $\mathrm{CB}$ and Chen WN: EBV up-regulates cytochrome $c$ through VDAC1 regulations and decreases the release of cytoplasmic $\mathrm{Ca}^{2+}$ in the NPC cell line. Cell Biol Int 36: 733-738, 2012.

6. Mishra A, Sullivan L and Caligiuri MA: Molecular pathways: interleukin-15 signaling in health and in cancer. Clin Cancer Res 20: 2044-2050, 2014.

7. Koh SJ, Kim JM, Kim IK, Ko SH and Kim JS: Anti-inflammatory mechanism of metformin and its effects in intestinal inflammation and colitis-associated colon cancer. J Gastroenterol Hepatol 29: 502-510, 2014.

8. Ohmi Y, Ohkawa Y, Tajima O, Sugiura Y, Furukawa K and Furukawa K: Ganglioside deficiency causes inflammation and neurodegeneration via the activation of complement system in the spinal cord. J Neuroinflammation 11: 61, 2014.

9. Nitta H, Wada Y, Kawano Y, et al: Enhancement of human cancer cell motility and invasiveness by anaphylatoxin C5a via aberrantly expressed C5a receptor (CD88). Clin Cancer Res 19: 2004-2013, 2013.

10. Nunez-Cruz S, Gimotty PA, Guerra MW, et al: Genetic and pharmacologic inhibition of complement impairs endothelial cell function and ablates ovarian cancer neovascularization. Neoplasia 14: 994-1004, 2012. 
11. Corrales L, Ajona D, Rafail S, et al: Anaphylatoxin C5a creates a favorable microenvironment for lung cancer progression. J Immunol 189: 4674-4683, 2012.

12. Xu Y, Shi Y, Yuan Q, et al: Epstein-Barr Virus encoded LMP1 regulates cyclin D1 promoter activity by nuclear EGFR and STAT3 in CNE1 cells. J Exp Clin Cancer Res 32: 90, 2013.

13. Liao Q,Zeng Z, Guo X, et al: LPLUNC1 suppresses IL-6-induced nasopharyngeal carcinoma cell proliferation via inhibiting the Stat 3 activation. Oncogene 33: 2098-2109, 2014.

14. Tsang CM, Cheung YC, Lui VW, et al: Berberine suppresses tumorigenicity and growth of nasopharyngeal carcinoma cells by inhibiting STAT3 activation induced by tumor associated fibroblasts. BMC Cancer 13: 619, 2013.

15. Pan Y, Zhou F, Zhang R and Claret FX: Stat3 inhibitor Stattic exhibits potent antitumor activity and induces chemo- and radio-sensitivity in nasopharyngeal carcinoma. PloS One 8: e54565, 2013.

16. Beltrao P, Albanese V, Kenner LR, et al: Systematic functional prioritization of protein posttranslational modifications. Cell 150 413-425, 2012

17. Linares JF, Duran A, Yajima T, Pasparakis M, Moscat J and Diaz-Meco MT: K63 polyubiquitination and activation of mTOR by the p62-TRAF6 complex in nutrient-activated cells. Mol Cell 51: 283-296, 2013

18. Pejanovic N, Hochrainer K, Liu T, Aerne BL, Soares MP and Anrather J: Regulation of nuclear factor $\kappa \mathrm{B}(\mathrm{NF}-\kappa \mathrm{B})$ transcriptional activity via p65 acetylation by the chaperonin containing TCP1 (CCT). PloS One 7: e42020, 2012.

19. Li T, Diner BA, Chen J and Cristea IM: Acetylation modulates cellular distribution and DNA sensing ability of interferon-inducible protein IFI16. Proc Natl Acad Sci USA 109: 10558-10563, 2012.

20. Xie J, Peng M, Guillemette S, et al: FANCJ/BACH1 acetylation at lysine 1249 regulates the DNA damage response. PLoS Genet 8: e1002786, 2012

21. Eckner R: p53-dependent growth arrest and induction of p21: a critical role for PCAF-mediated histone acetylation. Cell Cycle 11: 2591-2592, 2012.

22. Webster BR, Scott I, Han K, et al: Restricted mitochondrial protein acetylation initiates mitochondrial autophagy. J Cell Sci 126: 4843-4849, 2013.

23. Mandal T, Bhowmik A, Chatterjee A, Chatterjee U, Chatterjee S and Ghosh MK: Reduced phosphorylation of Stat3 at Ser-727 mediated by casein kinase 2 - protein phosphatase $2 \mathrm{~A}$ enhances Stat 3 Tyr-705 induced tumorigenic potential of glioma cells. Cell Signal 26: 1725-1734, 2014.

24. Zhao J, Gong AY, Zhou R, Liu J, Eischeid AN and Chen XM: Downregulation of PCAF by miR-181a/b provides feedback regulation to TNF- $\alpha$-induced transcription of proinflammatory genes in liver epithelial cells. J Immunol 188: 1266-1274, 2012.

25. Love IM, Sekaric P, Shi D, Grossman SR and Androphy EJ: The histone acetyltransferase PCAF regulates p21 transcription through stress-induced acetylation of histone H3. Cell Cycle 11: 2458-2466, 2012.
26. Ge X, Jin Q, Zhang F, Yan T and Zhai Q: PCAF acetylates $\{$ beta\}-catenin and improves its stability. Mol Biol Cell 20: 419-427, 2009.

27. Patel JH, Du Y, Ard PG, et al: The c-MYC oncoprotein is a substrate of the acetyltransferases hGCN5/PCAF and TIP60. Mol Cell Biol 24: 10826-10834, 2004.

28. Zhu LH, Sun LH, Hu YL, et al: PCAF impairs endometrial receptivity and embryo implantation by down-regulating $\beta 3$-integrin expression via HOXA10 acetylation. J Clin Endocrinol Metab 98: 4417-4428, 2013

29. Wang LX, Wang J, Qu TT, Zhang Y and Shen YF: Reversible acetylation of Lin28 mediated by PCAF and SIRT1. Biochim Biophys Acta 1843: 1188-1195, 2014.

30. Schlottmann S, Erkizan HV, Barber-Rotenberg JS, et al: Acetylation increases EWS-FLI1 DNA binding and transcriptional activity. Front Oncol 2: 107, 2012.

31. Wang CY, Yang SF, Wang Z, et al: PCAF acetylates Runx 2 and promotes osteoblast differentiation. J Bone Miner Metab 31: 381-389, 2013.

32. Rajendran R, Garva R, Ashour $\mathrm{H}$, et al: Acetylation mediated by the $\mathrm{p} 300 / \mathrm{CBP}$-associated factor determines cellular energy metabolic pathways in cancer. Int J Oncol 42: 1961-1972, 2013.

33. Zhou HB, Yin YF, Hu Y, et al: Suppression of vascular endothelial growth factor via siRNA interference modulates the biological behavior of human nasopharyngeal carcinoma cells. Jpn J Radiol 29: 615-622, 2011.

34. Kedjarune-Leggat U, Supaprutsakul C and Chotigeat W: Ultrasound treatment increases transfection efficiency of low molecular weight chitosan in fibroblasts but Not in KB cells. PloS One 9: e92076, 2014.

35. Qiu W, Zhang Y, Liu X, et al: Sublytic C5b-9 complexes induce proliferative changes of glomerular mesangial cells in rat Thy-1 nephritis through TRAF6-mediated PI3K-dependent Akt1 activation. J Pathol 226: 619-632, 2012.

36. Liu Y, Li Z, Wu L, et al: MiRNA-125a-5p: a regulator and predictor of gefitinib's effect on nasopharyngeal carcinoma. Cancer Cell Int 14: 24, 2014.

37. Di Paolo NC, Baldwin LK, Irons EE, Papayannopoulou T, Tomlinson S and Shayakhmetov DM: IL-1 $\alpha$ and complement cooperate in triggering local neutrophilic inflammation in response to adenovirus and eliminating virus-containing cells. PLoS Pathog 10: e1004035, 2014.

38. Hoth JJ, Wells JD, Jones SE, Yoza BK and McCall CE: Complement mediates a primed inflammatory response after traumatic lung injury. J Trauma Acute Care Surg 76: 601-609, 2014.

39. Hsiao JR, Jin YT, Tsai ST, Shiau AL, Wu CL and Su WC: Constitutive activation of STAT3 and STAT5 is present in the majority of nasopharyngeal carcinoma and correlates with better prognosis. Br J Cancer 89: 344-349, 2003.

40. Ma N, Kawanishi M, Hiraku Y, et al: Reactive nitrogen species-dependent DNA damage in EBV-associated nasopharyngeal carcinoma: the relation to STAT3 activation and EGFR expression. Int J Cancer 122: 2517-2525, 2008. 